\title{
EXPERIMENTAL FOUNDATIONS OF EXPLOSIVE NUCLEOSYNTHESIS
}

\author{
W. A. FOWLER \\ California Institute of Technology 106-38 \\ Pasadena, California 91125, U.S.A.
}

\begin{abstract}
Laboratory studies of nuclear reaction rates relevant to carbon, oxygen and silicon burning in explosive nucleosynthesis will be discussed. Emphasis will be placed on the role of excited nuclear states in the reactions involved.
\end{abstract}

In this paper I will give a short review of certain developments in nuclear astrophysics since my George Darwin lecture (Fowler [1]) late in 1973. It will not be necessary to discuss developments in hydrogen burning, helium burning or carbon-oxygen burning since these have been recently summarized in Fowler, Caughlan and Zimmerman [2]. Suffice it to say that the solar neutrino problem is still with us, helium burning rates are much better known than here-to-fore but more experimental work is needed on a number of $(\alpha, \gamma)$ and $(\alpha, n)$ reactions and there still remain the great uncertainties in the extrapolation of the ${ }^{12} \mathrm{C}+{ }^{12} \mathrm{C}$ and ${ }^{16} \mathrm{O}+{ }^{16} \mathrm{O}$ reactions to the astrophysically important energy range below the lowest energy at which experimental measurement has been possible.

Much progress has been made during the past two years in the nuclear laboratory in measurements on the cross-sections of those reactions which are thought to be relevant to quasistatic silicon burning in the pre-supernova stage of stellar evolution and to explosive nucleosynthesis during the supernovae stage. The motivation underlying the experimental research and the associated theoretical analysis is the belief that nuclear cross-sections and the derived reaction rates can serve as diagnostic tools in determining conditions of density, temperature, time scale, neutron or proton excess, and initial composition under which nucleosynthesis occurred. In this way, it will eventually be possible to make unique choices for the sites where nuclear processes produced the characteristic abundance distribution of the elements and their isotopes observed in the Sun, normal stars, peculiar stars, the interstellar medium, and the cosmic rays in our Galaxy.

Silicon burning under quasistatic or explosive conditions involves a reaction rate network which includes upwards of 500 reactions and their reverse reactions, involving both stable and radioactive nuclei, and considerably more if the reactions involving the low-lying excited states of these nuclei are counted separately (see Fig. 1 in Fowler [1]).
Figures 1 and 2 show the cross-section measurements and theoretical calculations (solid curves) for just two of the reactions recently studied at Caltech and elsewhere. The agreement with theoretical HauserFeshbach calculations is in general quite good,

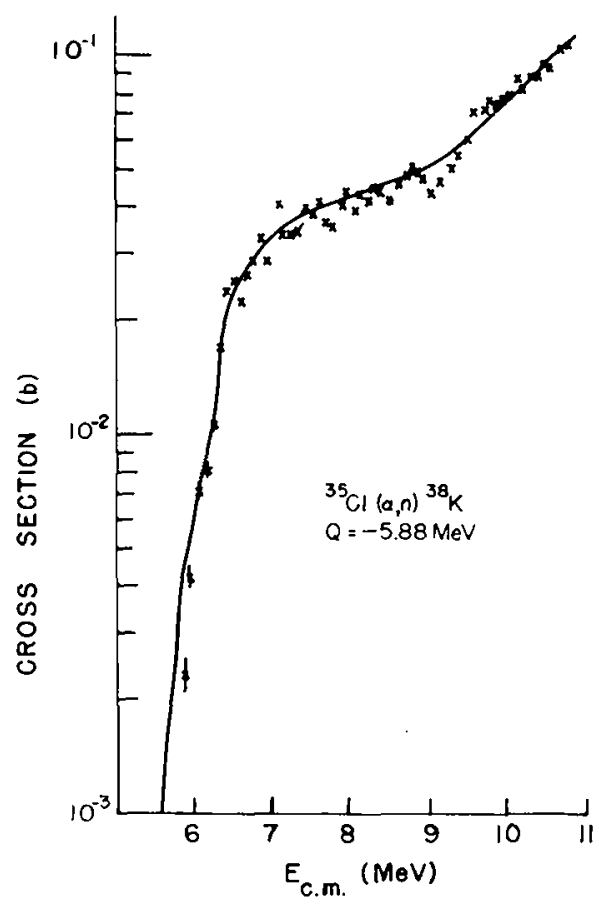

FiG. 1. - The cross-section for ${ }^{35} \mathrm{Cl}(\alpha, n){ }^{38} \mathrm{~K}$ from the ground state of ${ }^{35} \mathrm{Cl}$ to all bound states of ${ }^{38} \mathrm{~K}$ energetically permitted. Experimental points from Mann \& Kavanagh [5]. Hauser-Feshbach calculations.

especially at low energies where the theoretical results depend critically on the radius parameters used for the incident channel. A single set of parameters has been used in all the Hauser-Feshbach calculations. These are taken from table I of Michaud and Fowler [3]. The gamma-ray transmission functions used are discussed later in this paper. 


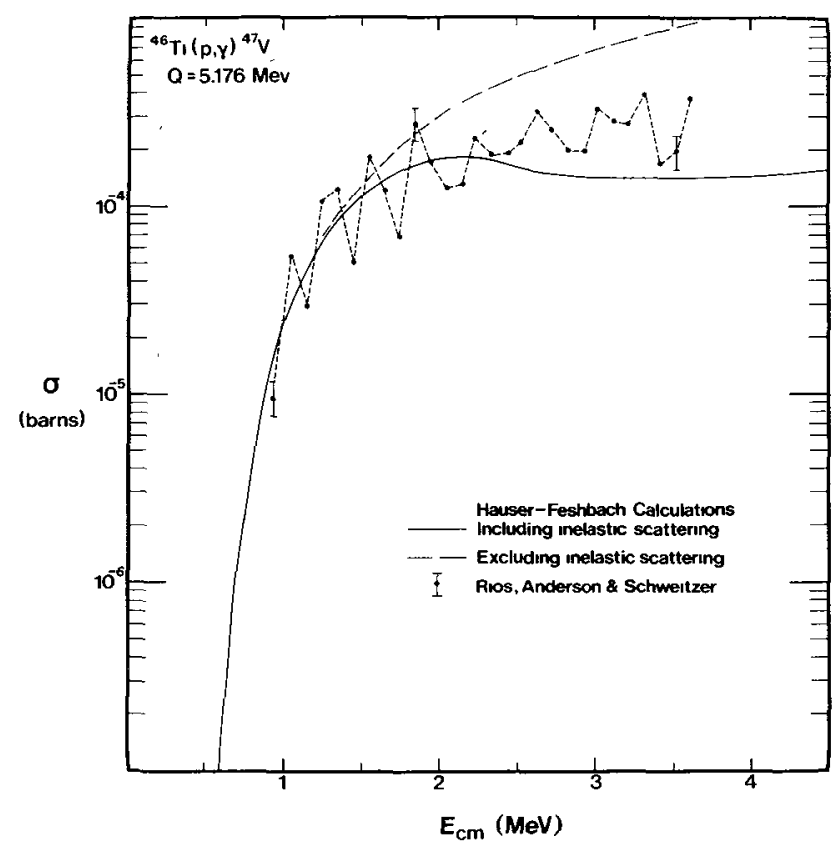

Fig. 2. - The cross-section for ${ }^{46} \mathrm{Ti}(\mathrm{p}, \gamma)^{47} \mathrm{~V}$ from the ground state of ${ }^{46} \mathrm{Ti}$ to all bound states of ${ }^{47} \mathrm{~V}$ energetically permitted, obtained by measuring positron annihilation radiation from ${ }^{47} \mathrm{~V}$. Experimental points from Rios, Anderson, and Schweitzer [10]. It is clear that the Hauser-Feshbach program must include excited states of the target nucleus, even though the agreement with experiment is still only fair at high energies.

The literature abounds with strengths and radius parameters to be used in the real and imaginary potentials necessary to calculate the transmission functions required in Hauser-Feshbach theory. In addition, numerous prescriptions have been put forward to correct the theory for channel-channel correlations, level-level correlations, width fluctuations and strong absorption, and to include the contribution of direct reactions.

Fortunately, the energy range of astrophysical interest is below the Coulomb barrier and direct reactions, in contrast to general expectations, have smaller cross-sections than those proceeding through the compound nucleus. This matter has been given considerable study and the results published by Switkowski, Wieland and Winther [4].

We are involved in a systematic attempt to determine empirically the run of Hauser-Feshbach parameters for the intermediate-mass nuclei in the range $20 \leqslant A \leqslant 80$. The results to date have been given in the thesis Comparison of Experimental cross-sections with the Hauser-Feshbach Model, by F. M. Mann and will be published soon (Mann and Kavanagh [5]). At the same time, we have pressed forward with use of the simplified theory described by Michaud and Fowler [3] and have modified it for correlations and strong absorption, following the prescription of Tepel, Hofmann and Weidenmuller [4]. This prescription preserves reciprocity but violates unitarity. However, the main error occurs in the evaluation of the elasticscattering cross-section. We are only interested in inelastic or reaction channels in astrophysical applications and here the errors are small. For example, in $\mathrm{a}(\mathrm{p}, \gamma)$ or $(\alpha, \gamma)$ reaction, the incident particle transmission function becomes equal to the sum over the many gamma-ray transitions near $E=1 \mathrm{MeV}$ for protons and near $E=3 \mathrm{MeV}$ for alpha particles, corresponding in both cases to temperatures in the range $1 \lesssim T_{9} \lesssim 2$. The error is a maximum in this case and $\sigma_{\gamma}$ (Unmodified HF) $=1.37 \sigma_{\gamma}$ (Exact HF) and $\sigma_{\gamma}$ (Modified HF) $=0.91 \sigma_{\gamma}$ (Exact HF). The exact Hauser-Feshbach calculation is a very good but hard-to-apply approximation given by Tepel et al. [6] which preserves unitarity. At the present state of the art these errors are both of marginal significance but there is some controversy involved concerning the other corrections and we intend to pursue the entire matter in the future.

The eventual aim is to produce a computer program for astrophysical reaction rates which can be made available to all astrophysicists in the form of computer tapes or cards. In the meantime, we are issuing a preliminary evaluation of cross-sections and reaction rates using our unmodified program for ease of comparison of experimental data with theory (Woosley, Fowler, Holmes and Zimmerman [7]). Systematic deviations from the unmodified theory will become apparent and realistic modifications will become available as this program is pursued. A systematic semiempirical theory of reaction rates is essential to further progress in nuclear astrophysics. It is impossible to measure all the reactions involved - there are too many of the reactions and shortlived radioactive nuclei and excited states cannot be used as targets. Measurement of reverse reactions and the use of reciprocity helps, but it is abundantly clear that some rates will be calculable only from theory which therefore must be as reliable and inclusive as possible.

A major problem has been the determination of gamma-radiation transmis-functions for use in Hauser-Feshbach calculations. Through the work of Woosley, Holmes, Fowler and Zimmerman [8] we have finally reached an empirical formulation fitted to measured $(\mathrm{n}, \gamma)$ cross-sections as shown in figure 3 ,

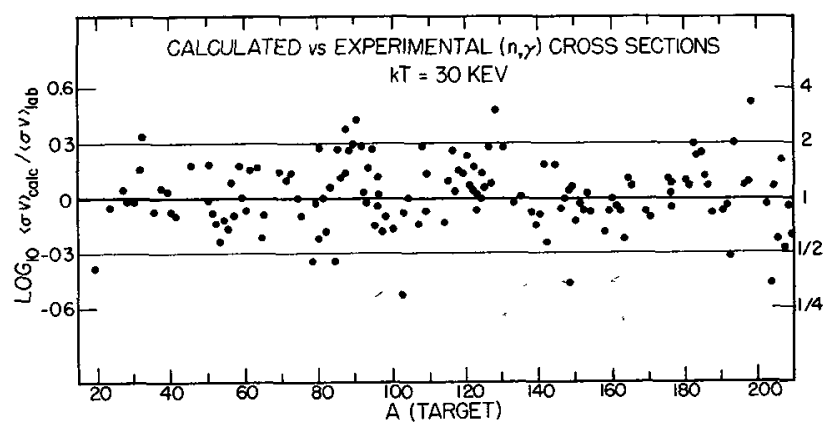

FIG. 3. - Calculated $(\mathrm{n}, \gamma)$ cross-sections vs thermally averaged experimental cross-sections at $k T=30 \mathrm{keV}$. The experimental data have been used to determine the free parameters in the gamma-ray transmission functions used in the calculations. 
and are now beginning to compare measured (p, $\gamma$ ) and $(\alpha, \gamma)$ cross-sections with this formulation. We will continue to make measurements and comparisons with theory in the hope that a formulation which will predict $(\mathrm{n}, \gamma),(\mathrm{p}, \gamma)$ and $(\alpha, \gamma)$ cross-sections with small error will emerge: Considerations concerning isotopic-spin selections rules must be included.

For low bombarding energies we find the following expression for the gamma-ray transmission function to be accurate in general to $\pm 50 \%$ :

$$
\begin{aligned}
T(\gamma) \approx & \frac{8 \times 10^{-9}}{A^{1 / 6} a^{2}}(B-\Delta)^{1 / 2} \times \\
& \times \exp \left[2 a^{1 / 2}(B-\Delta)^{1 / 2}+3 / a^{1 / 2}(B-\Delta)^{1 / 2}\right]
\end{aligned}
$$

with

$$
\Delta=\alpha A^{-1 / 2}-80 A^{-1} \mathrm{MeV}
$$

and

$$
\begin{aligned}
\alpha & =0 \text { for odd-odd compound nuclei } \\
& =11 \text { for even-odd compound nuclei } \\
& =22 \text { for even-even compound nuclei } \\
a & =0.0518 A^{1.2} \\
A & =\text { mass number of compound nucleus }
\end{aligned}
$$

$B=Q+E=$ energy release in the radiative capture reaction with $Q$ the reaction energy release and $E$ the center-of-momentum energy.

This expression replaces equation (23) of Michaud and Fowler [3]. It is high for self-conjugate compound nuclei and these cases will come under further extensive study since they are important in the reaction network of explosive nucleosynthesis. This expression must also be modified when open particle channels successfully compete with cascade gamma-ray transitions. This is discussed in detail in Woosley, Holmes, Fowler and Zimmerman [8] who include discrete and continuum transitions in their calculations.

Temperatures up to $T \sim 100^{10} \mathrm{~K}$ are of interest in nuclear astrophysics and it will thus be clear that excited states in reacting nuclei will be populated up to excitations of the order of $k T \sim 1 \mathrm{MeV}$. We have shown that the time for population to the equilibrium value is the order of the spontaneous lifetime of the state. These lifetimes are short for low-order multipole radiation transitions, e.g., $10^{-12} \mathrm{~s}$ even for a state with width $10^{-3} \mathrm{eV}$. The lifetimes are much shorter than even the shortest dynamical times for explosive nucleosynthesis which is $\sim 10^{-2} \mathrm{~s}$ for an initial density of $10^{10} \mathrm{~g} \mathrm{~cm}^{-3}$. Thus the equilibrium population is to be expected for all except isomeric states which must be inventoried as separate nuclear species in all calculations.

We have given much thought to the inherent symmetry necessary in the consideration of excited states of the target nucleus vis à vis excited states of the residual nucleus. Laboratory measurements yield, when required, the sum over transitions to all bound excited states of the residual nucleus and it is this sum which is relevant to reaction rates under stellar circumstances. The transition to an excited state in a residual nucleus is detected in the laboratory through a particle of lower kinetic energy than that to the ground state, and also through subsequent decay by gamma-ray emission or particle break-up. A radioactive groundstate makes it all easy.

It will be intuitively clear on grounds of symmetry that a simple sum over transitions from all excited states of target nuclei must appear in the overall reaction rate between the initial and final reactants when they exist in an equilibrium population under stellar circumstances even though one starts with an average rather an a sum over initial states. This is easily proven to be the case using the Hauser-Feshbach statistical theory, and leads to a considerable simplification when this theory is used to calculate overall effective cross-sections (Fowler [1]).

Figure 4 shows a comparison between theoretical and experimental rates for 48 reactions which have been recently measured in our laboratory and elsewhere, over a sufficient energy range and with enough precision to give astrophysical reaction rates with an uncertainty of less than $50 \%$. The comparison with theory will be discussed in what follows, but first we emphasize how little has been done $(48 / 500 \sim 10 \%)$ in spite of heroic efforts in the laboratory. Many more experimental measurements on, and theoretical analysis of the reactions of intermediate-mass nuclei with protons and alpha-particles are needed.

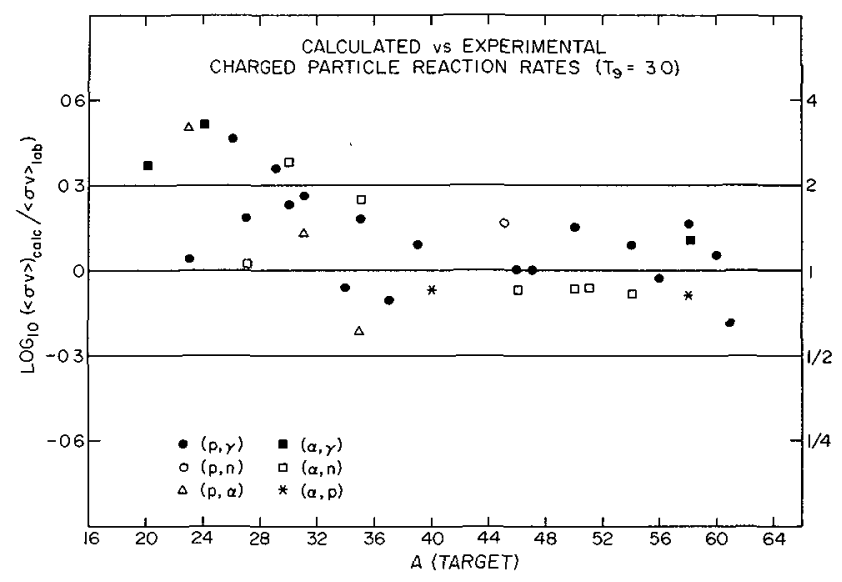

FIG. 4. - Calculated reaction rates vs experimental reaction rates at $T=3.0 \times 10^{9} \mathrm{~K}$. From Woosley, Holmes, Fowler \& Zimmerman [8].

It will be clear from figure 4 that a global approach using a Hauser-Feshbach program with a unique set of radius and potential parameters agrees closely with experiment for intermediate-mass targets above $A \sim 30$. For lower mass targets the calculated values are in general high. This is expected since the HauserFeshbach calculations give an average overall reso- 
nances. When levels are narrow and far apart, as can occur for low $A$, the stellar range of energies will usually be deficient in resonances. In a few cases where a strong resonance dominates the stellar range of energies the Hauser-Feshbach calculations will be low. This occurs in ${ }^{21} \mathrm{Ne}(\mathrm{p}, \gamma)$. In all these cases it is necessary to study the level structure of the compound nucleus in the range of interest either directly or by the use of other reactions. Interesting developments in this regard have been reported by Rolfs et al. [9].
In any case, a fairly accurate expression for gammaray transition functions is now available. Furthermore, the effect of excited states of target nuclei in stellar reaction rates is in principle understood and can be indirectly checked experimentally and can be incorporated economically into Hauser-Feshbach calculations. Reliable nuclear data are rapidly becoming available for use in realistic static and dynamic calculations of the presupernova and supernovae stages of stellar evolution.

\section{References}

[1] Fowler, W. A., Q. J. R. Astron. Soc. 15 (1974) 82.

[2] Fowler, W. A., Caughlan, G. R. and Zimmerman, F. A., Annu. Rev. Astron. Astrophys. 13 (1975) 69.

[3] Michaud, G. and Fowler, W. A., Phys. Rev. C2 (1970) 2041.

[4] Switkowski, Z. E., Wieland, R. M. and Winther, A., Phys. Rev. Lett. 33 (1974) 840.

[5] Mann, F. M. and KavanaGH, R. W. 1975, in press.

[6] Tepel, J. W., Hofmann, H. M. and Weidenmuller, H. A., Phys. lett. B 49 (1974) 1.
[7] Woosley, S. E., Fowler, W. A., Holmes, J. A. and ZimmerMaN, B. A., 1975, Orange Aid Preprint $n^{\circ} 422$ (available on request to William $A$. Fowler).

[8] Woosley, S. E., Holmes, J. A., Fowler, W. A. and ZIMMerMAN, B. A., 1976 in preparation.

[9] Rolfs, C., Rodney, W. S., Shapiro, M. H. and WinKLeR, H., Nucl. Phys. A 241 (1975) 460.

[10] Rios, C., Anderson, B. I. and Schweitzer, J. S., Nucl. Phys. A 236 (1974) 523. 